\title{
Clinical efficacy of MVD combined with PSR in the treatment of primary trigeminal neuralgia
}

\author{
GUANGYU ZHAO, XIAOJUN SUN, ZHEN ZHANG, HONGAN YANG, XIANGRONG ZHENG and BIN FENG
}

Department of Neurosurgery, Shandong Provincial Hospital, Jinan, Shandong 250021, P.R. China

Received August 21, 2019; Accepted February 4, 2020

DOI: $10.3892 /$ etm.2020.8871

\begin{abstract}
Clinical efficacy of microvascular decompression (MVD) combined with percutaneous stereotactic radiofrequency rhizotomy (PSR) in the treatment of primary trigeminal neuralgia was investigated. The medical records of 141 patients with primary trigeminal neuralgia admitted to Shandong Provincial Hospital from May 2011 to June 2013 were collected. Among them, 63 patients received MVD surgery and were set as group A, while the other 78 received MVD combined with PSR and were as group B. The efficacy and complication of the two treatment methods were compared. Multivariate logistic regression was performed to analyze the risk factors for treatment efficacy. The total effective rate was $96.15 \%$ in group $\mathrm{B}$, higher than that in group A $(88.89 \%)$, but the difference was not statistically significant $(\mathrm{P}>0.05)$. The complications in group B were statistically less than that in group A $(\mathrm{P}<0.05)$. Risk factors for the onset of primary trigeminal neuralgia included the degree of decompression, duration of disease, degree of compression, and clinical symptoms. Patients treated with MVD combined with PSR had a better quality of life and lower 5-year recurrence rate than patients treated with MVD (both $\mathrm{P}<0.05$ ). In conclusion, MVD combined with PSR treatment has good clinical efficacy in primary trigeminal neuralgia and low incidence of complications. The possible risk factors for the onset of primary trigeminal neuralgia include the degree of decompression, duration of disease, degree of compression, and clinical symptoms.
\end{abstract}

\section{Introduction}

Trigeminal neuralgia is a severe unilateral facial pain involving one or more branches of the trigeminal nerve $(1,2)$. It is usually unilateral, severe, transient, and recurrent, causing great pain to patients. As the disease progresses, patients may have difficulty

Correspondence to: Dr Bin Feng, Department of Neurosurgery, Shandong Provincial Hospital, 324 Jingwu Road, Jinan, Shandong 250021, P.R. China

E-mail: frboy8@163.com

Key words: MVD, PSR, primary trigeminal neuralgia eating, talking and maintaining facial hygiene in fear of triggering the pain, which has a profound impact on the quality of life $(1,3,4)$. Neuropathic pain is common in trigeminal neuralgia, peripheral neuropathy, and multiple sclerosis (5). Primary trigeminal neuralgia is caused by the compression of the trigeminal nerve by adjacent blood vessels (6), which is recurrent and very difficult to treat conservatively. Its unbearable severe pain has serious effects on the physical and psychological health of patients (7-9), so its treatment has become the focus of clinical research.

The current preferred surgical treatment for primary trigeminal neuralgia is microvascular decompression (MVD) (10). MVD, a minimally invasive interventional technique which is able to identify the pain nerve, can effectively isolate the responsible vessels compressing the roots of the trigeminal nerve and the brainstem so as to relieve the compression, and repair the pain nerve under the operating microscope to eradicate the trigeminal pain source. This minimally invasive technique completely retains the function of blood vessels and nerves, is an effective treatment for trigeminal neuralgia $(11,12)$. However, clinical data revealed that MVD is not effective for some patients, leaving poorly relieved pain and a large postoperative trauma (13). Percutaneous stereotactic radiofrequency rhizotomyroot (PSR) is also common in treating primary trigeminal neuralgia (14), suitable for cases like recurrent disease after surgery and failed identification of the responsible blood vessels by MVD. PSR only partially cuts off the sensory roots, so it can save the function of the patient's movement roots and relieve the patient's facial pain in a very effective manner (15). However, clinical records show that some patients treated with PSR are prone to a variety of complications and a poor prognosis (16).

To find a better treatment for primary trigeminal neuralgia, in this study, the clinical efficacy of MVD and MVD combined with PSR in primary trigeminal neuralgia was compared to confirm the effect of MVD and PSR on primary trigeminal neuralgia to provide reference for the treatment of this disease.

\section{Patients and methods}

General information. A prospective analysis was performed. A total of 141 patients with primary trigeminal neuralgia admitted into Shandong Provincial Hospital (Jinan, China) from May 2011 to June 2013 were collected. Among them, 63 patients (26 male and 37 female, aged 37.6 \pm 7.9 years) with 
MVD were studied as group A, while the other 78 (45 male and 33 female, aged $36.5 \pm 8.3$ years) with MVD combined with PSR were studied as group B.

Inclusion and exclusion criteria. Inclusion criteria: Patients diagnosed with primary trigeminal neuralgia according to the criteria of the International Headache Society (IHS); patients with no neurological deficits; patients with poor efficacy from drug treatment; patients with significant nerve compression; patient with vascular compression according to imaging examination. The present study was approved by the Ethics Committee of Shandong Provincial Hospital (SPH201104). All patients and their families signed the informed consent.

Exclusion criteria: Patients with cardiovascular and cerebrovascular diseases, malignant tumors, mental disorders, symptoms of secondary trigeminal neuralgia such as multiple sclerosis or tumor nerve compression symptoms; patients with surgery contraindications; pregnant or lactating women.

Treatment methods. All surgery procedures were performed by the same physician. Patients were generally anesthetized and placed in the prone position, with head down at approximately 15 degrees to expose the mastoid of the operating side at the highest level of the head. In the longitudinal incision in the hairline behind the ear, the retrosigmoid sinus approach was made to fully expose the posterior margin of the mastoid. A hole was drilled in the posterior occipital lacunae of the mastoid and was expanded by a rongeur to make a bone window of approximately 1.5 to $2.5 \mathrm{~cm}$ and the transverse sinus and sigmoid sinus were exposed. The mastoid air cell was closed by bone wax. The dura mater was given a ' $\perp$ ' cut and suspended. The subarachnoid space was opened under the microscope, and the cerebellar hemisphere was lightly pulled to the inner side with the brain plate. The arachnoid was sharply cut with a micro-scissor to identify the compressing vessels and isolate it from the initial segment of the trigeminal nerve root. The Teflon cotton was placed between the responsible vessels and the brain stem to decompress the blood vessels. 6 hours after the completion of MVD, PSR was performed to detect the presence of thick veins on the surgical site to compress the trigeminal nerve. The arachnoid band between the vein and the nerve or the brainstem was cut, and the veins were free. Isolation, distance or suspension were selected to decompress according to the type of venous compression (electrocoagulation shearing can be performed in the case of difficult separation), and then PSR is performed on the sensory nerves $1 / 3$ to $1 / 5$ of the posterior lateral of the trigeminal sensory root. Under the microscope, the trigeminal nerve root was carefully and sharply cut off. The operation is performed with caution. It is strictly required to avoid mechanical damage to blood vessels and nerves.

Postoperative treatment. The patient was treated with intravenous dexamethasone to avoid postoperative rejection. Routine examinations were performed after the surgery to monitor the recovery and the complications. If no abnormalities were observed, patients were allowed to discharge one week after the surgery.
Table I. Efficacy evaluation.

\begin{tabular}{ll}
\hline Response & Criteria \\
\hline Cured & $\begin{array}{l}\text { The pain completely disappeared and } \\
\text { no drug was needed. } \\
\text { The pain was relieved by 90\%, the } \\
\text { Marked response }\end{array}$ \\
$\begin{array}{l}\text { drug was used occasionally or in a } \\
\text { small dose. }\end{array}$ \\
The pain was relieved to a certain \\
degree, the dosage of the drug was \\
reduced by 50\%, or the multiple pain \\
turned into single pain. \\
The pain was not relieved.
\end{tabular}

Outcome measures. Main outcome measure: The postoperative surgical outcomes of the two groups were observed. The efficacy criteria are shown in Table I.

Secondary outcome measures: The incidence of postoperative complication in the two groups was observed; the risk factors affecting the efficacy were explored by the multivariate analysis; the quality of life after the treatment of the two groups was monitored; the 5-year recurrence rate of the two groups was observed.

Statistical methods. Statistical analysis was performed using SPSS 19.0 (Chicago SPSS Co., Ltd.), and the collected data were visualized using GraphPad Prism 7 (San Diego Graphpad Software Co., Ltd.). The count data were expressed with rate (\%) and compared by the Chi-square test. The measurement data were expressed with the mean \pm standard deviation (mean $\pm \mathrm{SD}$ ) and compared between two groups by the independent sample t-test. Logistic regression test was used for multivariate analysis. K-M survival curve was used to analyze the 5-year recurrence of patients. A statistical difference was recognized at $\mathrm{P}<0.05$.

\section{Results}

General clinical data of group A and group B. Group A (patients treated with MVD) and group B (patients treated with MVD combined with PSR) were not statistically different in age, sex, BMI $\left(\mathrm{kg} / \mathrm{m}^{2}\right)$, painful side, clinical symptoms, compressing vessels, compression degree, duration of disease, place of residence, smoking or drinking $(\mathrm{P}>0.05)$. Details are shown in Table II.

Evaluation of the efficacy in group $A$ and group $B$. The total effective rate was $88.89 \%$ in group $A$, lower than that in group B $(96.15 \%)$, but the difference was not statistically significant $(\mathrm{P}>0.05)$ (Table III).

The incidence of complications after treatment in group A and group $B$. The number of patients experiencing nausea and vomiting, peripheral facial paralysis, hearing loss, cerebrospinal fluid leakage, subcutaneous effusion in group A were $13,5,3,1$, and 3 , respectively, and the total incidence of 
Table II. General clinical data of group A and group B [n (\%)].

\begin{tabular}{|c|c|c|c|c|}
\hline Factors & Group A $(n=63)$ & Group B $(\mathrm{n}=78)$ & $\mathrm{t} / \chi^{2}$ value & P-value \\
\hline Sex & & & 3.760 & 0.053 \\
\hline Male & $26(41.27)$ & $45(57.69)$ & & \\
\hline Female & $37(58.73)$ & $33(42.31)$ & & \\
\hline Age (years) & $37.6 \pm 7.9$ & $36.5 \pm 8.3$ & 0.799 & 0.426 \\
\hline BMI $\left(\mathrm{kg} / \mathrm{m}^{2}\right)$ & $23.57 \pm 2.15$ & $24.07 \pm 2.13$ & 1.380 & 0.170 \\
\hline Painful side & & & 0.263 & 0.608 \\
\hline Left side & $32(50.79)$ & $43(55.13)$ & & \\
\hline Right side & $31(49.21)$ & $35(44.87)$ & & \\
\hline Clinical symptoms & & & 0.564 & 0.453 \\
\hline Typical & $34(53.97)$ & $47(60.26)$ & & \\
\hline Not typical & $29(46.03)$ & $31(39.74)$ & & \\
\hline Compressing vessels & & & 0.983 & 0.612 \\
\hline Artery compression & $18(28.57)$ & $17(21.79)$ & & \\
\hline Venous compression & $20(31.75)$ & $25(32.05)$ & & \\
\hline Mixed compression & $25(39.68)$ & $36(46.15)$ & & \\
\hline Compression degree & & & 0.017 & 0.897 \\
\hline Displaced & $30(44.44)$ & $38(42.31)$ & & \\
\hline Not displaced & $33(47.62)$ & $40(46.15)$ & & \\
\hline Duration of disease (years) & & & 5.425 & 0.994 \\
\hline$<5$ & $25(39.68)$ & $31(39.74)$ & & \\
\hline$\geq 5$ & $38(60.32)$ & $47(60.26)$ & & \\
\hline Place of residence & & & 0.101 & 0.751 \\
\hline Urban area & $29(46.03)$ & $38(48.72)$ & & \\
\hline Rural area & $34(53.97)$ & $40(51.28)$ & & \\
\hline Smoking & & & 0.015 & 0.901 \\
\hline Yes & $26(41.27)$ & $33(42.31)$ & & \\
\hline No & $37(58.73)$ & 45 (57.69) & & \\
\hline Drinking & & & 0.726 & 0.394 \\
\hline Yes & $23(36.51)$ & $34(43.59)$ & & \\
\hline No & $40(63.49)$ & $44(56.41)$ & & \\
\hline
\end{tabular}

Table III. Evaluation of the efficacy in group A and group B [n (\%)].

\begin{tabular}{lcccc}
\hline Response & Group A $(\mathrm{n}=63)$ & Group B $(\mathrm{n}=78)$ & $\chi^{2}$ value & P-value \\
\hline Cured & $38(60.32)$ & $48(61.54)$ & 0.022 & 0.883 \\
Marked response & $10(15.87)$ & $15(19.23)$ & 0.269 & 0.604 \\
Moderate response & $8(12.70)$ & $12(15.38)$ & 0.207 & 0.650 \\
No response & $7(11.11)$ & $3(3.85)$ & 2.792 & 0.095 \\
Total effective rate & $56(88.89)$ & $75(96.15)$ & 2.792 & 0.095 \\
\hline
\end{tabular}

adverse reaction in group A was $39.68 \%$, while the numbers in group B were 5, 3, 1, 0, and 1, respectively, and the total incidence of adverse reaction in group B was $12.82 \%$. The difference between the two groups in the adverse reactions was statistically significant $(\mathrm{P}<0.05)$. Additional details are shown in Table IV.
Univariate analysis of the efficacy. The two groups of patients were divided into the cured group $(n=86)$ and the not-cured group $(n=55)$ according to efficacy. The univariate analysis of the clinical data of the two groups demonstrated that the cured group and the not-cured group were not statistically different in sex, age, BMI $\left(\mathrm{kg} / \mathrm{m}^{2}\right)$, painful 
Table IV. The incidence of complications after treatment in group A and group B [n (\%)].

\begin{tabular}{|c|c|c|c|c|c|c|c|c|}
\hline Group & $\begin{array}{l}\text { Nausea and } \\
\text { vomiting }\end{array}$ & $\begin{array}{c}\text { Peripheral } \\
\text { facial paralysis }\end{array}$ & $\begin{array}{l}\text { Hearing } \\
\text { loss }\end{array}$ & $\begin{array}{l}\text { Cerebrospinal } \\
\text { fluid leakage }\end{array}$ & $\begin{array}{l}\text { Subcutaneous } \\
\text { effusion }\end{array}$ & Total & $\chi^{2}$ value & P-value \\
\hline Group A $(n=63)$ & $13(20.63)$ & $5(7.94)$ & $3(4.76)$ & $1(1.59)$ & $3(4.76)$ & $25(39.68)$ & 13.48 & $<0.001$ \\
\hline Group B $(n=78)$ & $5(6.41)$ & $3(3.85)$ & $1(1.28)$ & $0(0)$ & $1(1.28)$ & $10(12.82)$ & & \\
\hline
\end{tabular}

Table V. Univariate analysis.

\begin{tabular}{|c|c|c|c|c|}
\hline Factors & Cured group $(n=86)$ & Not-cured group $(n=55)$ & $\mathrm{t} / \chi^{2}$ value & P-value \\
\hline Sex & & & 0.010 & 0.919 \\
\hline Male & $32(37.21)$ & $20(36.36)$ & & \\
\hline Female & $54(62.79)$ & $35(63.64)$ & & \\
\hline Age (years) & $39.7 \pm 8.1$ & $38.5 \pm 8.3$ & 0.850 & 0.397 \\
\hline BMI $\left(\mathrm{kg} / \mathrm{m}^{2}\right)$ & $25.87 \pm 2.83$ & $26.07 \pm 1.78$ & 0.468 & 0.641 \\
\hline Painful side & & & 0.066 & 0.767 \\
\hline Left side & $41(47.67)$ & $25(45.45)$ & & \\
\hline Right side & $45(52.33)$ & $30(54.55)$ & & \\
\hline Clinical symptoms & & & 12.840 & $<0.001$ \\
\hline Typical & $62(72.09)$ & $23(41.82)$ & & \\
\hline Not typical & $24(27.91)$ & $32(58.18)$ & & \\
\hline Compressing vessels & & & 4.250 & 0.119 \\
\hline Artery compression & $27(31.40)$ & $14(25.45)$ & & \\
\hline Venous compression & $30(34.88)$ & $13(23.64)$ & & \\
\hline Mixed compression & $29(33.72)$ & $28(50.91)$ & & \\
\hline Compression degree & & & 13.600 & $<0.001$ \\
\hline Displaced & $29(33.72)$ & $36(65.45)$ & & \\
\hline Not displaced & $57(66.28)$ & $19(34.55)$ & & \\
\hline Decompression degree & & & 47.130 & $<0.001$ \\
\hline Full decompression & $74(86.05)$ & $16(29.09)$ & & \\
\hline Not-full decompression & $12(13.95)$ & $39(70.91)$ & & \\
\hline Duration of disease (years) & & & 16.510 & $<0.001$ \\
\hline$<5$ & $52(60.47)$ & $14(25.45)$ & & \\
\hline$\geq 5$ & $34(39.53)$ & $41(74.55)$ & & \\
\hline Place of residence & & & 0.299 & 0.585 \\
\hline Urban area & $46(53.49)$ & $32(58.18)$ & & \\
\hline Rural area & $40(46.51)$ & $23(41.82)$ & & \\
\hline Smoking & & & 1.033 & 0.309 \\
\hline Yes & $36(47.37)$ & $31(56.36)$ & & \\
\hline No & $50(52.63)$ & $24(43.64)$ & & \\
\hline Drinking & & & 0.211 & 0.646 \\
\hline Yes & $33(38.37)$ & $19(34.55)$ & & \\
\hline No & $53(61.63)$ & $36(65.45)$ & & \\
\hline
\end{tabular}

side, compressing vessels, smoking, drinking, and place of residence $(\mathrm{P}>0.05)$, but statistically different in the decompression degree, duration of disease, compression degree, and clinical symptoms $(\mathrm{P}<0.05)$. Additional details are shown in Tables V-VII.
Comparison of postoperative quality of life between group A and group $B$. The quality of life of patients at one year of treatment in group B was significantly better than that of patients in group B in terms of physiological role, overall health, physiological function, vitality, physical pain, mental health, 
Table VI. Assignments.

\begin{tabular}{ll}
\hline Factors & \multicolumn{1}{c}{ Assignment } \\
\hline Decompression degree & $\begin{array}{l}\text { Full decompression }=1 ; \\
\text { not-full decompression }=0\end{array}$ \\
Duration of disease & $<5$ years $=1 ; \geq 5$ years $=0$ \\
Compression degree & $>2$ times $=1 ; 2$ times $=0$ \\
Clinical symptoms & Typical $=0 ;$ not typical $=1$
\end{tabular}

emotional function, social function $(\mathrm{P}<0.05)$. More details are shown in Table VIII.

The 5-year recurrence rate in both groups. According to the statistics, all 141 patients and their families were successfully followed up. Five years after the surgery, 18 patients in group A had recurrent disease, with a recurrence rate of $28.57 \%$, and 10 patients in group B had recurrent disease, with a recurrence rate of $12.82 \%$. The figure of the 5-year recurrence of the two groups displayed that the 5-year recurrence rate of group $B$ was statistically lower than that of group $\mathrm{A}(\mathrm{P}=0.022)$ (Fig. 1).

\section{Discussion}

Primary trigeminal neuralgia is debilitating, characterized by unilateral facial pain following the sensory distribution of cranial nerve V (17). Most patients with long-term use of drugs can develop drug resistance and experience side effects (18), and thus need surgery. MVD is the most common surgery for primary trigeminal neuralgia, but it is limited by age, pain level, medical comorbidities, past surgery procedures, and patient preference $(19,20)$. Minimally invasive techniques such as PSR are designed to damage nerves for pain control $(21,22)$. To discover better treatments for primary trigeminal nerves, the application of MVD combined with PSR was investigated in this study.

This study found that MVD combined with PSR treatment had better efficacy than MVD alone. The study by Zeng et al (23) compared the efficacy of MVD combined with PSR and MVD for primary trigeminal neuralgia and found that MVD combined with PSR was better than MVD alone because the pain was completely eliminated soon after PSR combined with MVD, which is similar to the results of the present study. The study by Zeng et al only listed the pain relief rate of the patient for two years, but in this study, the follow-up time was extended and it was found that the recurrence rate of patients receiving MVD combined with PSR was significantly lower than that of patients receiving MVD alone. It was speculated that MVD combined with PSR can identify responsible blood vessels more accurately, treat patients with different symptoms and eliminate the pain more efficiently. The study by Du et al (24) employed percutaneous balloon compression of trigeminal ganglion for recurrent trigeminal neuralgia after MVD because they believed MVD does not achieve a $100 \%$ cure rate and it carries high risk and low success rate for recurrent MVD, suggesting that MVD treatment is not suitable for all the patients. MVD is

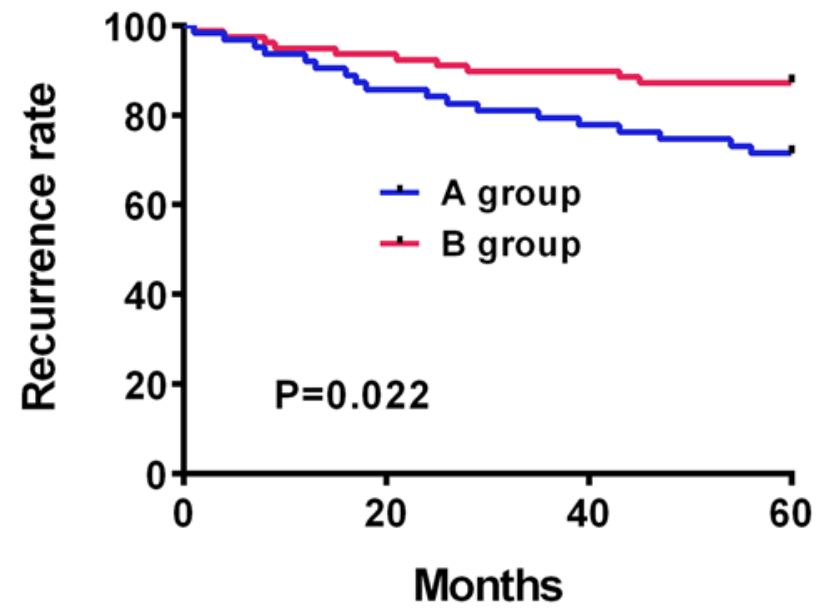

Figure 1. Five-year recurrence in two groups of patients. All patients underwent a 5-year follow-up, with a total recurrence rate of $19.86 \%$. The 5-year recurrence rate was $28.57 \%$ in group A, $12.82 \%$ in group B. The 5-year recurrence rate was lower in group $\mathrm{B}$ than that in group $\mathrm{A}(\mathrm{P}=0.022)$.

a non-destructive treatment. With identified pain nerve by medical equipment, MVD can relieve the compression of the roots of the trigeminal nerve and preserve the functions of blood vessels and nerves. PSR can remove the root nerve to stop the pain. MVD is not suitable for patients whose responsible vessels are not determined, besides, it causes large trauma. Considering different needs of different patients, it was speculated that MVD combined with PSR treatment can be applied to more patients to achieve better efficacy. The postoperative complications of the two treatments were compared and the results showed that MVD combined with PSR treatment had significantly fewer complications than MVD, indicating that MVD combined with PSR may be a better treatment for primary trigeminal neuralgia. A univariate analysis was performed on the efficacy of the two groups before the multivariate analysis and a statistical difference was found between the patients cured and not cured in the degree of decompression, duration of disease, degree of compression, and clinical symptoms. Multivariate analysis was then performed by logistic regression test and revealed that the decompression degree, duration of the disease, the compression degree, and the clinical symptoms were risk factors for the patient's poor efficacy. Such results suggest that more attention should be paid to select suitable treatment for patients with poor decompression and compression degree, long disease duration, and severe clinical symptoms to achieve better efficacy. The following comparison of quality of life scores between the two groups demonstrated that patients treated with MVD combined with PSR had a higher quality of life and a lower 5-year recurrence rate than patients treated with MVD alone. This further suggests that the efficacy of MVD combined with PSR is better.

In summary, MVD combined with PSR in the treatment of primary trigeminal neuralgia has better efficacy, fewer complications, higher quality of life of patients, and lower 5-year recurrence rate than MVD alone. The risk factors affecting the efficacy of patients include the decompression degree, duration of disease, compression degree, and clinical symptoms. 
Table VII. Multivariate analysis for factors affecting efficacy.

$95 \%$ C.I. of $\operatorname{EXP}(\mathrm{B})$

\begin{tabular}{lccccccc}
\cline { 6 - 8 } Factors & B & S.E. & Wals & Sig. & Exp (B) & Lower limit & Upper limit \\
\hline Duration of disease & 0.889 & 0.409 & 4.175 & 0.030 & 2.432 & 1.090 & 5.423 \\
Clinical symptoms & 3.132 & 0.895 & 2.238 & 0.000 & 6.917 & 3.964 & 13.498 \\
Compression degree & 1.172 & 0.347 & 11.431 & 0.001 & 3.299 & 1.637 & 6.371 \\
Decompression degree & 4.060 & 1.009 & 4.208 & 0.000 & 7.312 & 8.034 & 14.732
\end{tabular}

Table VIII. Comparison of postoperative quality of life between group A and group B.

\begin{tabular}{|c|c|c|c|c|}
\hline Quality of life score & Group B $(n=78)$ & Group A $(n=63)$ & $\mathrm{t}$ value & P-value \\
\hline Physiological role & $36.19 \pm 6.61^{\mathrm{a}}$ & $25.53 \pm 5.28$ & 8.068 & $<0.001$ \\
\hline Overall health & $39.62 \pm 8.34^{\mathrm{a}}$ & $28.39 \pm 8.70$ & 5.967 & $<0.001$ \\
\hline Physiological function & $42.52 \pm 11.09^{\mathrm{a}}$ & $33.65 \pm 10.11$ & 3.785 & $<0.001$ \\
\hline Vitality & $44.45 \pm 6.91^{\mathrm{a}}$ & $35.98 \pm 6.67$ & 5.647 & $<0.001$ \\
\hline Physical pain & $46.94 \pm 8.81^{\mathrm{a}}$ & $37.05 \pm 8.01$ & 5.318 & $<0.001$ \\
\hline Mental health & $48.18 \pm 7.28^{\mathrm{a}}$ & $36.72 \pm 7.23$ & 7.152 & $<0.001$ \\
\hline Emotional function & $43.42 \pm 8.78^{a}$ & $32.97 \pm 8.19$ & 5.573 & $<0.001$ \\
\hline Social function & $43.72 \pm 7.89^{\mathrm{a}}$ & $36.87 \pm 7.16$ & 4.117 & $<0.001$ \\
\hline
\end{tabular}

${ }^{\mathrm{a}} \mathrm{P}<0.05$ when compared with group A after treatment.

\section{Acknowledgements}

Not applicable.

\section{Funding}

No funding was received.

\section{Availability of data and materials}

The datasets used and/or analyzed during the present study are available from the corresponding author on reasonable request.

\section{Authors' contributions}

GZ, XS and BF conceived and designed the study. GZ, ZZ, $\mathrm{HY}$ and $\mathrm{XZ}$ were responsible for the collection, analysis and interpretation of the data. GZ drafted the manuscript. GZ and $\mathrm{BF}$ revised the manuscript critically for important intellectual content. GZ wrote the manuscript. All authors read and approved the final manuscript.

\section{Ethics approval and consent to participate}

The study was approved by the Ethics Committee of Shandong Provincial Hospital (Jinan, China). Signed informed consents were obtained from the patients and/or the guardians.

\section{Patient consent for publication}

Not applicable.

\section{Competing interests}

The authors declare that they have no competing interests.

\section{References}

1. Moore D, Chong MS, Shetty A and Zakrzewska JM: A systematic review of rescue analgesic strategies in acute exacerbations of primary trigeminal neuralgia. $\mathrm{Br} \mathbf{J}$ Anaesth 123: e385-e396, 2019.

2. Zhang YP, Wang Y, Xia WG and Song AQ: Triple Puncture for primary trigeminal neuralgia: A randomized clinical trial. Curr Med Sci 39: 638-644, 2019.

3. $\mathrm{Hu} \mathrm{H}$, Chen L, Ma R, Gao H and Fang J: Acupuncture for primary trigeminal neuralgia: A systematic review and PRISMA-compliant meta-analysis. Complement Ther Clin Pract 34: 254-267, 2019.

4. Pokhrel D, Sood S, McClinton C, Saleh H, Badkul R, Jiang H, Stepp T, Camarata P and Wang F: Linac-based stereotactic radiosurgery (SRS) in the treatment of refractory trigeminal neuralgia: Detailed description of SRS procedure and reported clinical outcomes. J Appl Clin Med Phys 18: 136-143, 2017.

5. Qin ZL, Yang LQ, Li N, Yue JN, Wu BS, Tang YZ, Guo YN, Lai GH and Ni JX: Clinical study of cerebrospinal fluid neuropeptides in patients with primary trigeminal neuralgia. Clin Neurol Neurosurg 143: 111-115, 2016.

6. Montano N, Conforti G, Di Bonaventura R, Meglio M, Fernandez E and Papacci F: Advances in diagnosis and treatment of trigeminal neuralgia. Ther Clin Risk Manag 11: 289-299, 2015.

7. Wolf A and Kondziolka D: Trigeminal neuralgia and other facial neuralgias. Prog Neurol Surg 34: 273-278, 2019. 
8. Marchetti M, Pinzi V, De Martin E, Ghielmetti F and Fariselli L: Radiosurgery for trigeminal neuralgia: The state of art. Neurol Sci 40 (Suppl 1): 153-157, 2019.

9. Ding Y, Li H, Hong T, Zhu Y, Yao P and Zhou G: Combination of pulsed radiofrequency with continuous radiofrequency thermocoagulation at low temperature improves efficacy and safety in V2/V3 primary trigeminal neuralgia. Pain Physician 21: E545-E553, 2018.

10. Chen ZW, Ma JB, Xie KY, Huang B, Yao M, Fei Y and Zhang L: A study of the relations of foramen rotundum structure direction and the approach of percutaneous puncturing of radiofrequency thermocoagulation for treating V2 of primary trigeminal neuralgia. Zhonghua Yi Xue Za Zhi 98: 436-439, 2018 (In Chinese).

11. Liang C, Wang XY, Li J, Zhang M and Liu HF: Modified backward avulsion used in the treatment of third-branch primary trigeminal neuralgia. J Craniofac Surg 29: e87-e90, 2018.

12. Mistry AM, Niesner KJ, Lake WB, Forbes JA, Shannon CN, Kasl RA, Konrad PE and Neimat JS: Neurovascular compression at the root entry zone correlates with trigeminal neuralgia and early microvascular decompression outcome. World Neurosurg 95: 208-213, 2016.

13. Ma S, Agarwalla PK, van Loveren HR and Agazzi S: Successful microvascular decompression for trigeminal neuralgia secondary to a persistent trigeminal artery. Oper Neurosurg (Hagerstown) 16: 18-22, 2019.

14. Breeze RE and Craig DB: Microvascular decompression for trigeminal neuralgia: A durable, noncompressive technique using teflon secured with mini clip. Oper Neurosurg (Hagerstown) 16 : 580-582, 2019.

15. Wang Y, Yang Q, Cao D, Seminowicz D, Remeniuk B, Gao L and Zhang M: Correlation between nerve atrophy, brain grey matter volume and pain severity in patients with primary trigeminal neuralgia. Cephalalgia 39: 515-525, 2019.

16. Anichini G, Iqbal M, Rafiq NM, Ironside JW and Kamel M Sacrificing the superior petrosal vein during microvascular decompression. Is it safe? Learning the hard way. Case report and review of literature. Surg Neurol Int 7 (Suppl 14): S415-S420, 2016.
17. Hussain MA, Konteas A, Sunderland G, Franceschini P, Byrne P, Osman-Farah J and Eldridge P: Re-exploration of microvascular decompression in recurrent trigeminal neuralgia and intraoperative management options. World Neurosurg 117: e67-e74, 2018.

18. Kang IH, Park BJ, Park CK, Malla HP, Lee SH and Rhee BA A clinical analysis of secondary surgery in trigeminal neuralgia patients who failed prior treatment. J Korean Neurosurg Soc 59: 637-642, 2016

19. Wang DD, Raygor KP, Cage TA, Ward MM, Westcott S, Barbaro NM and Chang EF: Prospective comparison of long-term pain relief rates after first-time microvascular decompression and stereotactic radiosurgery for trigeminal neuralgia. J Neurosurg 128: 68-77, 2018.

20. Romanelli P, Conti A, Bianchi L, Bergantin A, Martinotti A and Beltramo G: Image-guided robotic radiosurgery for trigeminal neuralgia. Neurosurgery 83: 1023-1030, 2018.

21. Chen JN, Yu WH, Du HG, Jiang L, Dong XQ and Cao J: Prospective comparison of redo microvascular decompression and percutaneous balloon compression as primary surgery for recurrent trigeminal neuralgia. J Korean Neurosurg Soc 61: 747-752, 2018.

22. Zhang D, Meng Y, Hai H, Yu XT and Ma YW: Radial extracorporeal shock wave therapy in an individual with primary trigeminal neuralgia: A case report and literature review. Am J Phys Med Rehabil 97: e42-e45, 2018.

23. Zeng YJ, Zhang H, Yu S, Zhang W and Sun XC: Efficacy and safety of microvascular decompression and gamma knife surgery treatments for patients with primary trigeminal neuralgia: A prospective study. World Neurosurg 116: e113-e117, 2018.

24. Du Y, Yang D, Dong X, Du Q, Wang H and Yu W: Percutaneous balloon compression (PBC) of trigeminal ganglion for recurrent trigeminal neuralgia after microvascular decompression (MVD). Ir J Med Sci 184: 745-751, 2015. International (CC BY-NC-ND 4.0) License. 